\title{
ANALGESIA IN CHILDREN: IS THERE STILL A NEED FOR NOVEL APPROVED MEDICATIONS?
}

\section{Christoph Beuter \& Mariëlle Eerdekens}

\section{Background and aims}

For decades, medications have been used to treat paediatric patients without explicit regulatory approval. The European-Medicines-Agency (EMA) performed an assessment of paediatric needs for drug development between 2001 and 2007. Pain was identified as area lacking sufficient approved treatments (Doc.Ref.:EMEA/ CHMP/189220/2005). This may have been the result of a lack of development but also of market withdrawals of approved analgesics such as codein and metamizole for paediatric use in many countries. We assessed how the paediatric regulation, enacted in 2007 influenced the availability of authorized analgesics in paediatric patients.

\section{Methods}

We selected a number of commonly used analgesics in the paediatric population and verified their approved age ranges for use in their Summary-of-Drug-Characteristics (SMPC) published for Germany. We also checked the ongoing Pediatric-Investigational-Plans (PIPs) for analgesics and their potential to cover identified gaps.

Table 1: Approved Analgesics commonly used in the paediatric population
\begin{tabular}{|cc|cc|}
\hline \multicolumn{2}{|c|}{ Treatment of severe pain } & Treatment of moderate to severe pain \\
\hline Analgesic & Minimum age & Analgesic & Minimum age \\
\hline Morphine $^{*}$ & neonates & Codeine & 12 years \\
\hline Piritramid $^{* * *}$ & nd $^{* *}$ & Tramadol & 1 year \\
\hline Fentanyl & 2 years & Treatment of mild to moderate pain \\
\hline Hydromorphone & 1 year & Paracetamol & Term neonates \\
\hline Oxycodone & 12 years & lbuprofen & 3 months \\
\hline Metamizol & 3 months & Diclofenac & 6 years \\
\hline
\end{tabular}

Analgesics commonly used in the paediatric population and their approved age ranges for use. Information obtained from the German Summaries of Medicinal Product Characteristics (SMPCs) obtained from www.rote-liste.de. Only analgesics for systemic use were analysed.

*special precautions to be taken for infants below 1 year, ${ }^{* *}$ no minimum age defined, ***Used in combination products only

Table 2: Metrics for PIPs in the pain area

\begin{tabular}{|cc|}
\hline PIP Waiver granted & 32 \\
\hline Approved PIPs & 13 \\
\hline PIPs refused & 1 \\
\hline PIPs completed & 1 \\
\hline PIPs discontinued/ suspended & 2 \\
\hline
\end{tabular}

http://www.ema.europa.eu). Date of analysis: 22.Mar.2018

Table 3: Analgesics under investigation in the paediatric population

\begin{tabular}{|c|cc|}
\hline Pain Condition* $^{*}$ & Analgesic & Age range \\
\hline \multirow{4}{*}{ Acute pain } & Fentanyl & $\begin{array}{c}\text { Birth }-<2 \text { years (i.v. solution) } \\
2 \text { years }-<18 \text { years (plaster) }\end{array}$ \\
\cline { 2 - 3 } & Morphine & Birth $-<6$ months \\
\cline { 2 - 3 } & Tapentadol & Birth $-<18$ years \\
\cline { 2 - 3 } & Paracetamol ${ }^{* *}$ & Preterm $-<28$ days \\
\hline \multirow{2}{*}{$\begin{array}{c}\text { Chronic pain } \\
\text { Acute procedural } \\
\text { pain }\end{array}$} & Gabapentin & 3 months $-<18$ years \\
\cline { 2 - 3 } & Tapentadol & Birth $-<18$ years \\
\cline { 2 - 3 } & Gethoxyflurane & Birth $-<1$ year \\
\hline
\end{tabular}

Approved PIPs in the pain area that were not completed or discontinued/ suspended were assessed. Analgesics/medications used as analgesics mentioned in these PIPs are listed. (Source: http://www. ema.europa.eu). Date of analysis: 22.Mar.2018

*as given in the repective PIP; **original condition is given as moderate pain

\section{Results}

The availability of approved analgesics decreases with age (Tab.1). There is a lack of approved analgesics for treatment of moderate and severe pain in paediatric patients aged below 1 year (Tab.1). Overall few PIPs investigating analgesics are underway (Tab.2). Most of these PIPs investigate if the age range of approved analgesics in acute pain conditions can be extended (Tab.3).

The approval status of analgesics in Germany in Q1 2017 was used as basis for our analysis. The approval status of analgesics in other EUCountries may differ and a more limited number of approved alternatives may be available.

Tapentadol is the only novel analgesic developed for the entire paediatric population (Tab.3). This pediatric development plan is directly linked to the entry of tapentadol in the market for adults at the time that the paediatric regulation was enacted. However tapentadol's unique characteristics render it well suited for use across the entire paediatric population. It has a predictable pharmacokinetic profile, without active metabolites (Fig.1) and multiple elimination pathways via glucuronidation and sulfatation. The positive results of the PIP for acute pain, facilitated the recent approval of tapentadol for the treatment of acute pain in paediatric patients as of 2 years of age.

\section{Conclusions}

1. The access to approved analgesics is still limited for paediatric patients - especially for the very young.

2. Novel analgesics medications are still needed.

3. Only few PIPs addressing the lack of authorized pain treatments covering all paediatric age groups are currently progressed.

4. Tapentadol is the only novel analgesic medication investigated for its efficacy and safety in acute and chronic pain across the entire paediatric population.

5. Tapentadol has been approved for the treatment of acute pain in paeditric patients aged from 2 to less than 18 years old.

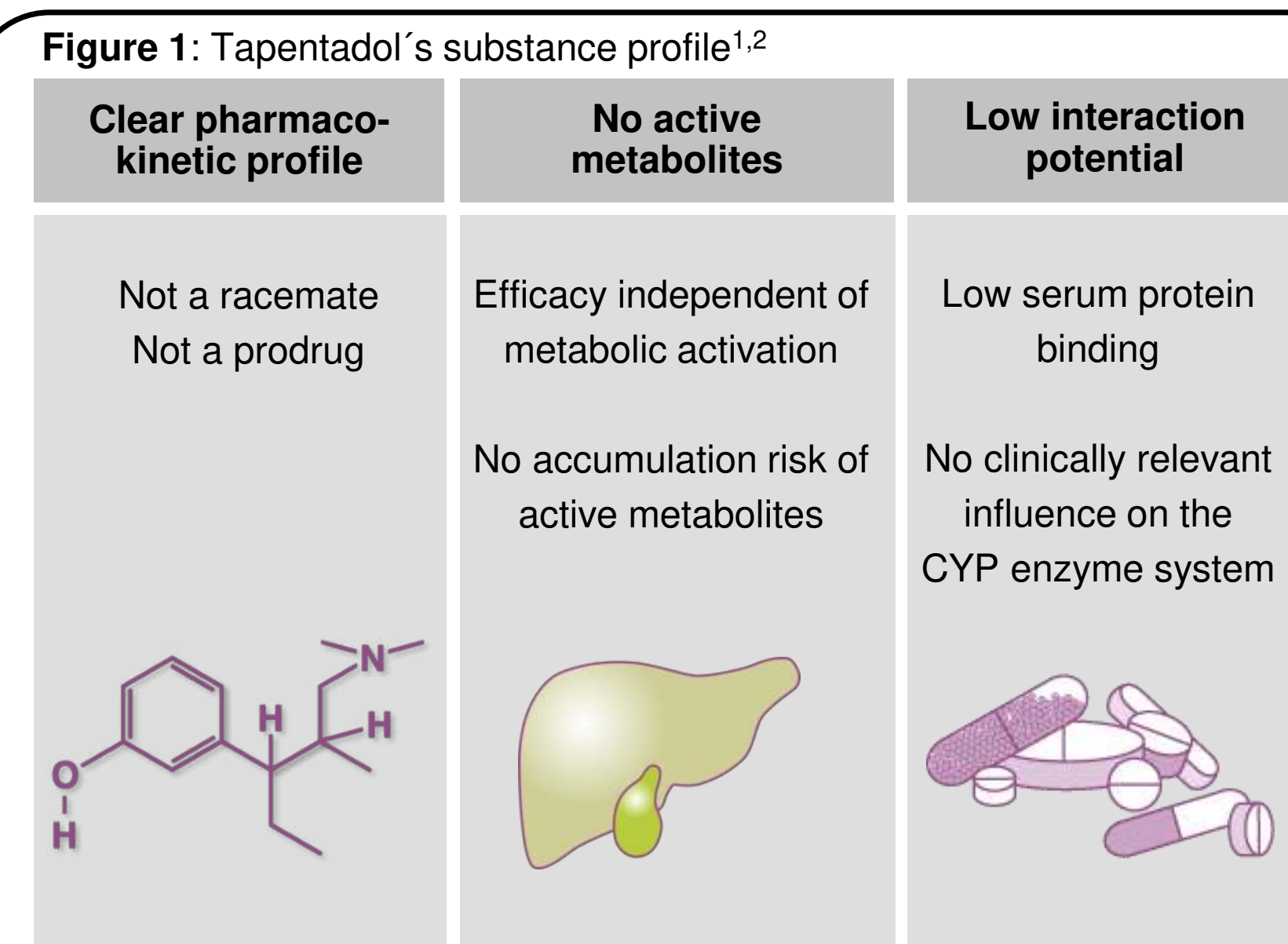

Tapentadol has a favourable pharmacokinetic profile with low interaction potential (ease of use). 